\title{
Shape accommodation of a rotating embedded crystal via a new variational formulation
}

\author{
JEAN E. TAYLOR ${ }^{\dagger}$ \\ Courant Institute, NYU, New York, NY 10012, USA \\ JOHN W. CAHN \\ National Institute of Standards \& Technology, Gaithersburg, MD 20899-8555, USA \\ Present address: University of Washington, Seattle, WA 98195-1560, USA
}

[Received 16 September 2006]

\begin{abstract}
We use an expanded variational approach based on dissipation to study the motion of the boundary of a non-circular cylindrical, and thus essentially 2-dimensional, crystalline grain of arbitrary cross-section enclosed in another grain of the same material under conditions where the normal grain boundary motion is coupled to relative tangential motion of the grains along the grain boundary. Coupling leads to relative rotation of the crystal axes of the two grains and requires shape accommodation; we assume that the necessary mass transport for shape accommodation is by diffusion confined to the grain boundary. We include the recently discovered fact that different modes of coupling with different coupling factors are crystallographically possible and do occur in molecular dynamic simulations.

Several results are deduced from the equations we derive. When there is a mirror plane symmetry to the crystal's initial shape, this symmetry plane remains and rotates at half the rate of rotation of the enclosed crystal's crystal axes relative to the surrounding crystal's axes. Sliding along the grain boundary is essential for shape change: when there is no sliding, we show that, although the inner crystal rotates and shrinks, its shape remains the same. If the cross-section of the enclosed crystal is large with a large aspect ratio and diffusion is relatively slow, then the rotation rate is initially diffusion controlled and very slow compared to nearly circular cylindrical crystals of the same cross-sectional area. In this case, and when sliding is relatively easy, the resulting normal motion is essentially mobility-controlled motion by weighted mean curvature; when sliding is intermediate in difficulty between mobility and diffusion, we have instead sliding-controlled motion by weighted mean curvature. As crystals become rounder and smaller in cross-section, all the kinetic factors become involved.
\end{abstract}

\section{Introduction}

\subsection{Overview}

We employ a new variational method to formulate and study a free boundary problem with many interacting, possibly non-linear, kinetic effects in which the available free energy and its dissipations can be formulated. The method is an extension of a variational approach for curvature driven flows [2, 6, 13]. The free boundary problem arises from the discovery that a moving grain boundary, which is an interface between two crystals of the same material, shears the material it traverses [4, 11]. This

\footnotetext{
†-mail: jtaylor@cims.nyu.edu

‡E-mail: cahnj@u.washington.edu
} 

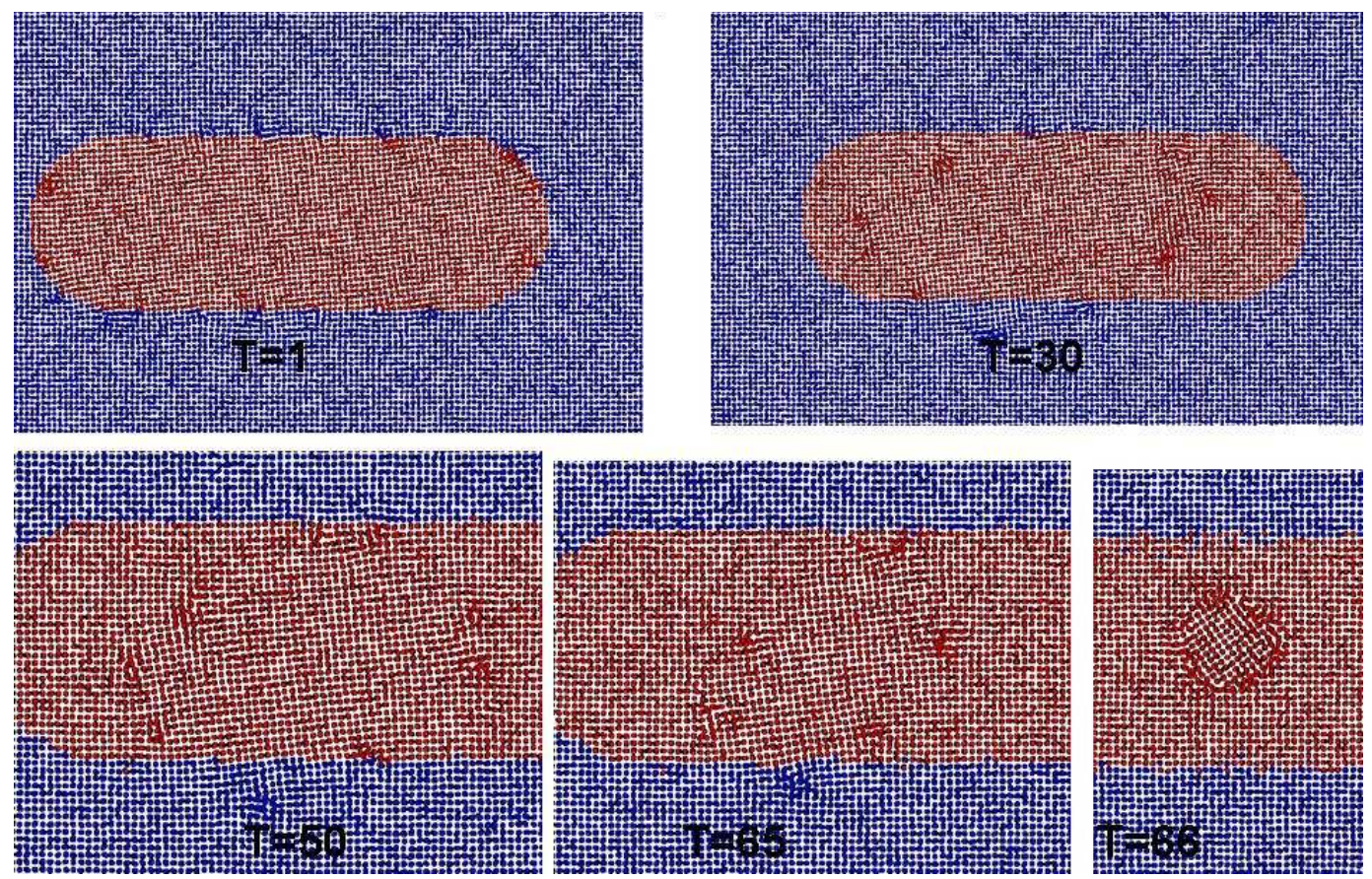

FIG. 1. Time snapshots from an unpublished 3D molecular simulation by S. G. Srinivasan in circa 2000 [10] of the shrinking of an enclosed grain which is initially an elliptical cylinder with a small misorientation between the grains. Dislocations are centered on the disturbances seen in the pattern and they collectively mark the grain boundary. The top left-hand figure shows the cross-section of the initial bicrystal, shortly after the start of the simulation $(T=1)$. As the enclosed grain shrinks the axis of the cylinder rotates, the misorientation between the grains increases to more than $30^{\circ}$, and the enclosed grain becomes rounder.

causes the axes of a shrinking crystal embedded in another crystal to rotate [5] (see Figure 1), and in general will require material transport for shape accommodation. In this paper we assume that the mechanism for the material transport is by diffusion confined to the grain boundary. We thus study grain boundary motion which incorporates coupling via a misorientation-dependent multivalued coupling factor $\beta$, shape accommodation via surface diffusion, and dynamics that include mobility and sliding. The diffusion along the surface is solely of the material required for shape accommodation in rotation; the flux is proportional to the rotation rate and does not directly involve the curvature, so there is no Laplacian of curvature anywhere in the motion laws we derive. Indeed, because there is no difference in density or composition between the two crystals, there cannot be any curvature-driven surface diffusion. The grain boundary motion laws we deduce form a new type of geometric crystal growth [14].

The understanding of the static and dynamic properties of interfaces between two crystalline solids is of great importance for many materials problems, but poses many difficulties. For dynamic properties it had long been assumed that the study of motion of grain boundaries in a system of just one chemical component would be simpler than general crystal growth. The realization that grain boundary motion is coupled to shear and that this leads to grain rotation is a complicating factor. In this paper as in our previous paper [5], the problem is made simpler by studying special bicrystals in which there are no triple junctions and only tilt boundaries- that is, in studying 
embedded cylindrical grains, where the cylinder axis coincides with a crystallographic axis common to both crystals. The previous paper considered only isotropic circular cylindrical grains. The major complication we address in this paper is the mass redistribution needed for a non-circular cylinder; we also consider other issues such as non-linear mobility.

We derive various results in Section 5. Among them are: If the grain shape has a plane of mirror symmetry, then in a coordinate system fixed to the axes of either grain that mirror plane rotates at half the rate of the axes of the other crystal. Motion with a variety of rates is seen to arise with coupling, not just motion by weighted curvature with mobility as the coefficient, as happens without coupling. In particular, one might have motion proportional to weighted mean curvature but with the coefficient proportional to the sliding coefficient and inversely proportional to the square of the coupling coefficient. If diffusion and sliding are difficult enough, then for nearly circular crystals $v_{\mathbf{n}}$ can have entirely diffusion-controlled motion, proportional to $1 / r^{3}$ rather than $1 / r$. See equations (23)- 26 . We end with a section on some obvious open problems.

\subsection{Choice of problem and assumptions}

As in the previous paper [5] we work entirely in two dimensions. We consider only two crystals, $A$ and $B$, also called grains, with $B$ embedded in $A$ and the interface curve $C$ between them referred to as a grain boundary. We think of $B$ as being the cross-section of a cylinder stretching to infinity and thus continue to use the language of three dimensions (we think of $\gamma$ as the surface free energy per unit area of the cylinder rather than the line free energy of the curve $C$, the grain boundary is everywhere a tilt grain boundary because the rotation axis is parallel to the cylinder axis, etc.) The crystals differ only by a rotation of their axes. Only two angles are needed to characterize an element of the boundary, the angle $\theta$ of misorientation between the crystals, and the "inclination" angle $\alpha$ of the directed tangent to some specified directed line. We assume that the motion is driven entirely by the reduction in the total free energy $\int_{C(t)} \gamma d s$, where $\gamma$ is a given positive real-valued function of $\alpha$ and $\theta$.

At various inclinations $\alpha$ and all $\theta$, a set of "ideal" coupling factors can be computed from a simple model of the possible crystallographic ways that atoms can be rearranged as they move from one crystal to the other. These describe simple shears which couple possible tangential velocities $V_{\|}$, of the lattice of $B$ relative to that of $A$, to the normal grain boundary velocity $v_{\mathbf{n}}$ of $A$ growing into $B$. Thus we assume we are given one or more continuous scalar coupling functions $\beta_{i}(\theta, \alpha)$, $i=1, \ldots, s$, with $s \geqslant 1$, where with pure coupling, $V_{\|}=\beta_{i} v_{\mathbf{n}}$ for some $i$. These functions are shown as the two curves in Figure 2 with Mode I as $i=1$ and Mode II as $i=2$. Our sign convention is that if the normal points up from $A$ below to $B$ above and the normal velocity is positive (up), then the tangential velocity is positive when $B$ moves to the right relative to $A$.

The coupling observed in simulations at low to intermediate temperatures with certain fixed $\alpha$ are seen as points in Figure 2 and fit this model with high accuracy, following either $\beta_{1}$ or $\beta_{2}$ [3]. We will call the coupling "ideal" if there is such agreement between simulation and the geometric model. (In the appendix, there is a compilation of some other data on coupling obtained through simulation.) Furthermore, if the $\beta_{i}$ are in fact the ideal coupling factors for all $\alpha$ and $\theta$, then each $\beta_{i}$ should be independent of $\alpha$, an assumption we will sometimes make in this paper. The variational formulation we introduce in this paper allows not only for the choice of which $\beta_{i}$ to use at each time but also for the possibility of different choices of $i$ being made at different points on the curve. We know of no examples where making different choices at different points is optimal. 


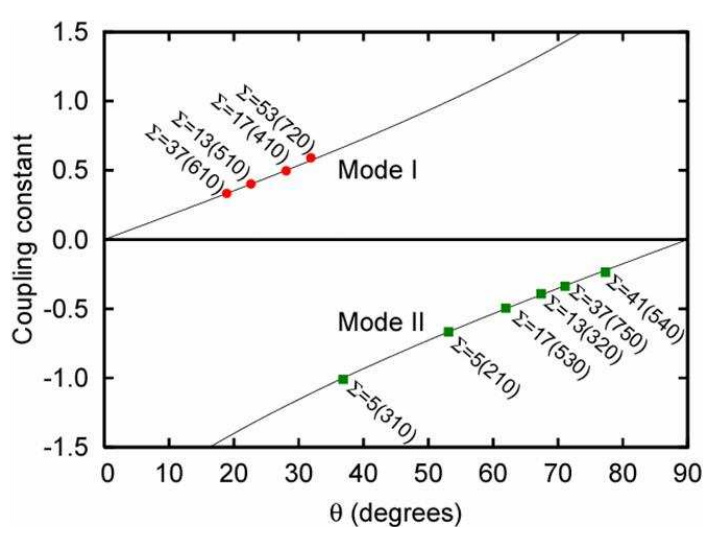

FIG. 2. Misorientation dependence of $\beta$ (Yuri Mishin data). Data from MD simulations for symmetric boundaries (constant $\alpha=0$ ), published in [4], demonstrating the excellent fit to the two coupling functions $\beta_{1}(\theta, 0)$ and $\beta_{2}(\theta, 0)$, as discussed in the appendix, and the fact that the system chooses to jump from one $\beta$ to the other. The misorientation is characterized by $\theta$.

At higher temperatures some rigid body sliding is also seen along the boundary. The magnitude and direction of sliding along the boundary is defined as the difference between that predicted from whichever coupling function $\beta_{i}$ applies and the tangential motion observed, i.e. $\beta_{i} v_{\mathbf{n}}-V_{\|}$. If a linear response is assumed, sliding is governed by a given sliding coefficient $S$, depending on $\theta$ and $\alpha$. Otherwise, the non-linearity is described by a function $\mathcal{S}$, which should be zero or increasing and have the same sign as its argument.

Motion of atoms into and out of the lattices, whether by dislocation climb for low angle boundaries or by what is often called attachment-detachment kinetics for higher angle boundaries, entails a dissipation. For linear kinetics its rate is governed by a given positive mobility constant $M_{i}$ which depends on the geometric mechanism as specified by the coupling factor $\beta_{i}$; in general, mobility may be a non-linear function $\mathcal{M}_{i}$ of the driving force. For each $i, \mathcal{M}_{i}$ should be increasing and have the same sign as its argument. We do NOT usually assume that $M_{i}$ is independent of $\alpha$ or $\theta$ and indeed know that it cannot be for some intervals of $\theta$. In fact, for flat interfaces, by Theorem 1 , $\beta_{1}$ is chosen over $\beta_{2}$ if $M_{1} \beta_{1}^{2}-M_{2} \beta_{2}^{2}>0$, and the locus of misorientations $\theta$ where $\beta_{1}$ switches to $\beta_{2}$ is sometimes known to depend on $\alpha$ for reasons of symmetry; see the appendix.

We assume that the rigidity of the grains is high enough for us to neglect any elastic distortion of grains so that the rotating grain undergoes pure Euclidian rigid body motion. This is a reasonable assumption, since the energy of any significant elastic distortion is large compared to the boundary energies in the system and elastic distortion would become huge with any significant rotation of nonround crystals. As a result, $\theta$ at any instant is the same all along the grain boundary (and in the interior), and a non-round crystal that rotates will no longer fit into its previous hole without shape accommodation. Observe that no accommodation problem would be encountered if the "orientation" of a grain could change without a rigid rotation of the material of the grain and its crystal axes. For example, if magnetostriction is ignored, no shape accommodation arises when the direction of magnetization of a ferromagnetic domain rotates independent of any rotation of the crystal axes. Computational models which do not account for shape accommodation, because "orientation" is treated as an order parameter [15], are more appropriate to such problems than to rotating crystalline grains.

We will assume that the redistributions, to accomplish the shape changes that are required by the rotation, occur by lateral diffusion along the grain boundary ("surface" diffusion). For linear 


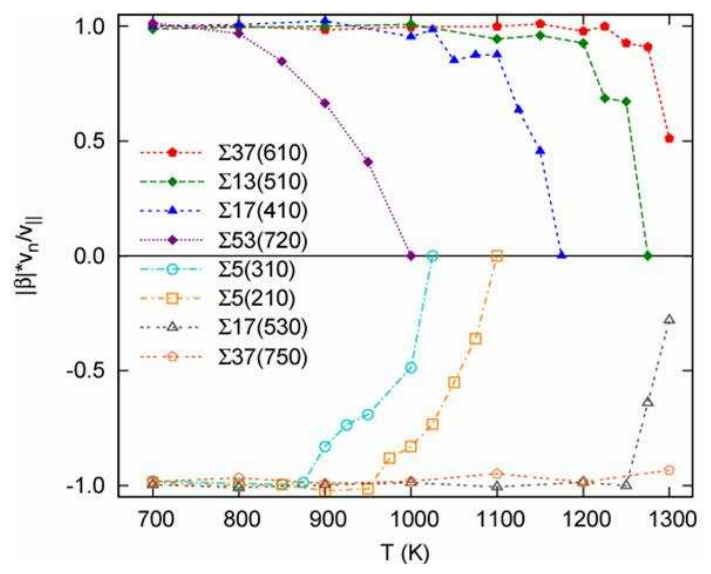

FIG. 3. Signed fraction of coupled motion versus $T$. Data from MD simulations on flat symmetric grain boundaries (fixed $\alpha$ ) at higher temperatures. Sliding is indicated by the fact that as temperature increases $\left|v_{n} / V_{\|}\right|$is first constant (no sliding), then decreasing (partial sliding), then zero (total sliding); the temperature at which sliding begins to be significant is a function of $\theta$.

diffusion, the rate is controlled by a diffusion coefficient $D$; for non-linear, a function $\mathcal{D}$ is given, which should be increasing and have the same sign as its argument. Locally the material is assumed to deposit smoothly and continuously on each grain at the grain boundary.

For simplicity, we make some assumptions on the $\alpha$-dependence of $\gamma$ and $\beta_{i}$. We assume that for each value of $\theta, \gamma$ is a twice continuously differentiable function of $\alpha$ and the equilibrium crystal shape for each $\theta$ (computed via the Wulff construction using $\gamma$, i.e. $W=\{x: x \cdot(\cos \alpha, \sin \alpha) \leqslant$ $\gamma(\alpha)$ for all $\alpha$ \} has no corners. We also assume that $\gamma^{\prime}:=\partial \gamma / \partial \theta$ exists for every $\alpha$ except at $\theta=0$. For much of the paper, we assume that each $\beta_{i}$ does not depend on $\alpha$. We assume that $M, S$, and $D$ (or their non-linear analogs) all depend continuously on $\alpha$.

Other limitations are that we consider only a fixed center of rotation. We also use a fixed temperature; it is worth noting that $M_{i}$ and $S$ depend strongly on this temperature (Figure 3 ).

\subsection{Comparison with earlier work}

In [5] we discussed coupled motion with planar grain boundaries, where the motion is driven by an applied stress. We also discussed motion with circular grain boundaries and linear kinetics where everything was independent of $\alpha$; here coupling produced rotation of crystal axes, with the motion laws

$$
\frac{\mathrm{d} r}{\mathrm{~d} t}=-M\left(\frac{\gamma-\beta \gamma^{\prime}}{r}\right), \quad r \frac{\mathrm{d} \theta}{\mathrm{d} t}=-\beta \frac{\mathrm{d} r}{\mathrm{~d} t}-S \frac{\gamma^{\prime}}{r} .
$$

In this paper, we study shape accommodation for non-circular grain boundaries and include non-linear kinetics and anisotropy. The new variational formulation is given for the linear case in (8) and for the general nonlinear in (9). The resulting equations for the linear case are (18) and (19), and for the non-linear case, (20) and (21).

In [5], where we studied circular crystals whose lattices have square symmetry, we knew by symmetry that the coupling would have to change sign between $\theta=0$ and $\pi / 2$ and mistakenly assumed that $\beta$ was a continuous, single-valued function of $\theta$ (we ignored any dependence on $\alpha$ 
in that paper). We now know that for such crystals there are at least four geometrically determined functions for $\beta$, but only two, one positive and one negative, are found to occur for $0<\theta<\pi / 2$ (which can be taken to be the entire range of misorientation, by symmetry). In addition [5] contained some sign errors: equation (22) there should begin with $v_{\|}=r \mathrm{~d} \theta / \mathrm{d} t$, not with $-v_{\|}=r \mathrm{~d} \theta / \mathrm{d} t$, and equation (30) there should be correspondingly corrected for signs.

In comparing the results of this paper with the previous paper [5], observe that what was called there $v_{\|}$is called here $V_{\|}$, and likewise for $V_{\perp}$. The lower-case letters $v_{\|}$and $v_{\perp}$ are reserved here for the velocities divided by $\dot{\theta}$ (see $\sqrt{6}$ ).

\section{Variational formulations involving mobility}

\section{$2.1 \quad$ Linear problems}

There are several equivalent ways to derive the law of motion for the normal velocity $v$ of a curve $C$, in the case where the velocity is assumed proportional, via a mobility factor $M$, to the driving force:

(i) If we write $\mathrm{d}(F+W) / \mathrm{d} t$, the rate change of free energy $F$ plus reversible work $W$ done, in the form $\int_{C}\left(-X v_{\mathbf{n}}+Y\right) \mathrm{d} s$ (so $X$ is the coefficient of $v_{\mathbf{n}}$ and $Y$ is everything else), the driving force for moving the grain boundary is defined as $X$. Assuming linear kinetics with mobility $M$, we set

$$
v_{\mathbf{n}}=M X .
$$

Since we do not consider applied stresses in this paper, we take $\mathrm{d} W / \mathrm{d} t$ to be zero.

(ii) We could instead add a term $\int_{C} \frac{1}{2 M} v_{\mathbf{n}}^{2} \mathrm{~d} s$ to $\int_{C}\left(-X v_{\mathbf{n}}+Y\right) \mathrm{d} s$ and then minimize with respect to $v_{\mathbf{n}}$. This yields equation (2) by a different route.

This is more than a mathematical trick. Approach (i) can be thought of as a surface version of the Lagrange equation for massless particles with a Rayleigh dissipation function $\mathcal{F}$. Consider particles with positions $\left\{q_{j}\right\}$, masses $\left\{m_{j}\right\}$ and velocities $\left\{\dot{q}_{j}\right\}$. The internal energy $E$ is assumed to depend only on $\left\{q_{j}\right\}$, and the kinetic energy $T$ is $\frac{1}{2} m_{j} \dot{q}_{j}^{2}$. Then with Lagrangian $L=T-E$, the Lagrange equations are ([7])

$$
\frac{\mathrm{d}}{\mathrm{d} t}\left(\frac{\partial L}{\partial \dot{q}_{j}}\right)-\frac{\partial L}{\partial q_{j}}+\frac{\partial \mathcal{F}}{\partial \dot{q}_{j}}=0 .
$$

We set $\mathcal{F}=\int_{0}^{\dot{q}_{j}} \frac{1}{M} u \mathrm{~d} u=\frac{1}{2} \frac{1}{M} \dot{q}_{j}^{2}$ and effectively assume all $m_{j}=0$ (that is, we assume there is no "mass" or momentum associated to the interface), thereby obtaining

$$
\dot{q}_{j}=-M \frac{\partial E}{\partial q_{j}},
$$

the particle version of equation (2). Approach (ii) can now be identified as finding critical points of $\mathcal{F}+\mathrm{d} E / \mathrm{d} t$, again assuming $T=0$. Although it is a variational principle, it is not Hamilton's principle, which involves variation over a time interval and yields an extra, unwanted, term when there is dissipation.

The dissipation function is $\mathcal{F}$; the dissipation rate under these assumptions is $2 \mathcal{F}$. The dissipation rate is NOT the dissipation function, and the dissipation rate will look even less like the dissipation function with dissipation functions corresponding to non-linear kinetics, as discussed below.

These ideas will be further discussed in [12]. 


\subsection{Extension to non-linear mobility}

More generally, for non-linear mobility, we assume we have a mobility function $\mathcal{M}$ and $v_{\mathbf{n}}=\mathcal{M}(X)$ for the driving force $X$. The dissipation function $\mathcal{F}$ should be $\int_{C} \int_{0}^{v_{\mathbf{n}}} \mathcal{M}^{-1}(u) \mathrm{d} u \mathrm{~d} s$. The dissipation rate is $v \partial \mathcal{F} / \partial v$. All the approaches still apply and result in $v_{\mathbf{n}}=\mathcal{M}(X)$.

In extending this approach to additional sources of dissipation, the key is to identify them and to express them as an overall dissipation function.

\section{Variational formulation for coupled motion with diffusional shape accommodation}

We propose here an expansion of the variational approach in which one minimizes the sum of the rate of energy increase and the rates of dissipation due to grain boundary motion and relative grain rotation. After fixing some notation we derive the rate for each energy change and the dissipation functions.

\subsection{Geometric preliminaries}

We consider a point $P$ on a smooth 2D grain boundary $C_{o}$ between grains $A$ and $B$ at time $t$. We will consider only the case where $C_{o}$ can be written in polar coordinates from its center of rotation $O$,

$$
\overline{P-O}=R(t, \phi)(\cos \phi, \sin \phi) \text {. }
$$

In this paper, we assume $O$ is fixed (e.g., by reason of symmetry). Let $\mathbf{n}$ be the normal direction of the grain boundary at $P$ in $C_{o}$, pointing from grain $A$ to grain $B$, and the tangential direction, chosen to be rotated clockwise from $\mathbf{n}$ by $90^{\circ}$.

Because we assume there is no elastic distortion, at any given time the orientation $\theta_{A}$ of the lattice of $A$ is the same at all points of $A$ and the orientation $\theta_{B}$ of the lattice of $B$ is the same throughout $B$. Since a rotation of the whole system leaves the system invariant, only the misorientation $\theta=\theta_{B}-\theta_{A}$ matters and is constant at each time. We may without loss of generality choose $\dot{\theta}_{A}$ as we wish. Although it might seem most natural to choose $\dot{\theta}_{A}=0$, so that $B$ rotates inside a fixed crystal, it is more convenient to choose $\dot{\theta}_{A}=-\dot{\theta}_{B}=-\dot{\theta} / 2$.

We define a set of local velocities at $P$. In general, when a non-circular cylindrical grain rotates, the rate of relative translation of the crystals at $P$ has to include a normal component as well as a tangential component. Let the vector $\mathbf{V}_{B \mid A}^{\text {lat }}(P)$ be the local velocity of the lattice of crystal $B$ as seen from a coordinate system imbedded in the lattice of crystal $A\left(\mathbf{V}_{B \mid A}^{\mathrm{lat}}(P)=-\mathbf{V}_{A \mid B}^{\mathrm{lat}}(P)\right)$. It is specified by a rotation rate $\mathrm{d} \theta / \mathrm{d} t$ about the center $\mathbf{O}$. Let $V_{\|}$be the local rate of relative tangential translation of the crystal B (into which $\mathbf{n}$ points), positive if in the direction $\mathbf{t}$. Then $V_{\|}(P):=$ $\mathbf{V}_{B \mid A}^{\text {lat }} \cdot \mathbf{t}$, and $V_{\perp}(P):=\mathbf{V}_{B \mid A}^{\text {lat }} \cdot \mathbf{n}$. The rate of volume increase per unit grain boundary area at $P$, which has to be removed by an accommodation process such as surface diffusion, is given by $V_{\perp}$. Let $v_{A}$ be the normal velocity of the grain boundary, relative to the lattice of $A$ and positive if in the direction of $\mathbf{n}$, and $v_{B}$ be the normal velocity of the grain boundary, relative to the lattice of $B$ and again positive if in the direction of $\mathbf{n}$; then $V_{\perp}=v_{A}-v_{B}$. We also define a mean normal velocity of the grain boundary as $v_{\mathbf{n}}:=\frac{1}{2}\left(v_{A}+v_{B}\right)$.

All of these local velocities can then be specified in terms of the relative rotation rate $\dot{\theta}$, and $R$ and its partial derivatives $\dot{R}=\partial R / \partial t$ and $R^{\prime}=\partial R / \partial \phi$. In particular, 


$$
\begin{aligned}
\mathbf{V}_{B \mid A}^{\text {lat }}(P) & =\dot{\theta} R(-\sin \phi, \cos \phi), \\
v_{A} & =-\dot{R} R / \sqrt{R^{2}+R^{\prime 2}}+R R^{\prime} \dot{\theta} /\left(2 \sqrt{R^{2}+R^{\prime 2}}\right), \\
v_{B} & =-\dot{R} R / \sqrt{R^{2}+R^{\prime 2}}-R R^{\prime} \dot{\theta} /\left(2 \sqrt{R^{2}+R^{\prime 2}}\right) .
\end{aligned}
$$

Thus

$$
\begin{aligned}
& V_{\perp}=R R^{\prime} \dot{\theta} / \sqrt{R^{2}+R^{\prime 2}}, \\
& V_{\|}=R^{2} \dot{\theta} / \sqrt{R^{2}+R^{\prime 2}}, \\
& v_{\mathbf{n}}=-\dot{R} R /\left(\sqrt{R^{2}+R^{\prime 2}}\right) .
\end{aligned}
$$

Observe that indeed the volume which has to be accommodated over an angular increment $\mathrm{d} \phi$ at $P$ per unit time and per unit cylinder height is $\dot{\theta} R R^{\prime} \mathrm{d} \phi=\dot{\theta} R R^{\prime} / \sqrt{R^{2}+R^{\prime 2}} \mathrm{~d} s=V_{\perp} \mathrm{d} s$, where $\mathrm{d} s$ is the arc increment $\sqrt{R^{2}+R^{\prime 2}} \mathrm{~d} \phi$.

The surface divergence of the diffusional flux $J$ along the grain boundary (volume per unit time across a unit length of the grain boundary) is $-V_{\perp}=v_{A}-v_{B}$, the rate of volume removal (accumulation) required to make up the difference between $v_{A}$ and $v_{B}$. In polar coordinates about the center of rotation of $B$ this is, using (4),

$$
\frac{R R^{\prime} \dot{\theta}}{\sqrt{R^{2}+R^{\prime 2}}}=\frac{\partial J}{\partial s}
$$

which can be integrated by inspection to give a remarkably simple result for $J$ in terms of $R, \dot{\theta}$, and a mean square radius $\left(\overline{R^{2}}\right)=\int_{C_{o}} R^{2} \mathrm{~d} s /$ length $\left(C_{o}\right)$ :

$$
J=\frac{1}{2}\left(R^{2}-\left(\overline{R^{2}}\right) \dot{\theta} .\right.
$$

(The integration constant is $\left(\overline{R^{2}}\right)$, which satisfies the conservation condition $\int J \mathrm{~d} s=0=$ $\left.\int_{C_{o}}\left(R^{2}-\left(\overline{R^{2}}\right)\right) \mathrm{d} s.\right)$

It is convenient to isolate the factors of $\dot{\theta}$. We therefore define

$$
v_{\perp}=\frac{V_{\perp}}{\dot{\theta}}, \quad v_{\|}=\frac{V_{\|}}{\dot{\theta}}, \quad j=\frac{J_{\perp}}{\dot{\theta}} .
$$

Each of these is purely geometrical, depending only on $R$ and $R^{\prime}$.

Finally, for completeness and for establishing the sign convention for $\kappa_{\gamma}$, the weighted curvature, we include its formula in these coordinates:

$$
\kappa_{\gamma}=\left(R^{2}+R^{\prime 2}\right)^{-3 / 2}\left(R^{2}+2 R^{\prime 2}-R R^{\prime \prime}\right)\left(\gamma+\frac{\partial^{2} \gamma}{\partial \alpha^{2}}\right) .
$$

\subsection{The changes in the total free energy}

The total free energy is the integral of $\gamma$. The rate of change of surface free energy under motion by normal velocity $v_{\mathbf{n}}$ (in this paper, the normal $\mathbf{n}$ points into the enclosed crystal $B$ ) is $-\int_{C} \kappa_{\gamma} v_{\mathbf{n}} \mathrm{d} s$ where $\kappa_{\gamma}$ is given in equation 7 . For a rotating crystal, writing $\gamma^{\prime}$ for $\frac{\partial \gamma}{\partial \theta}(\alpha, \theta)$, the rate of surface energy change becomes

$$
\int_{C_{o}}\left(\gamma^{\prime} \dot{\theta}-\kappa_{\gamma} v_{\mathbf{n}}\right) \mathrm{d} s .
$$


One way of obtaining this result is as a limit of time steps. Let the grain boundary be the curve $C_{o}$. After a time step $\Delta t$ we consider a comparison crystal shape with boundary $C$ and misorientation $\theta+\Delta \theta$. The average rate of surface energy change is

$$
\frac{1}{\Delta t}\left(\int_{C} \gamma\left(\mathbf{n}_{C}, \theta+\Delta \theta\right) \mathrm{d} s-\int_{C_{o}} \gamma\left(\mathbf{n}_{C_{o}}, \theta\right) \mathrm{d} s\right)
$$

Since we are assuming $C_{o}$ is smooth, we may assume that in the reference lattice for $A$, the image of $C$ under rotation by $\theta_{A}^{-1}$ is the image of $C_{o}$ under the normal velocity $v_{A}$ run for time $\Delta t$ and take the limit as $\Delta t$ approaches zero. Similarly, in the reference lattice for $B$, the image of $C$ under the rotation $\theta_{B}^{-1}$ is the image of $C_{o}$ under the normal velocity $v_{B}$ run for time $\Delta t$. There is a potential issue here, because $v_{A}$ and $v_{B}$ are different. But the curve $C$ is the same curve in each case, so the energy change should be the same in both coordinate systems, and the same as when we use $v_{\mathbf{n}}$ on $C$.

\subsection{The dissipation rates}

3.3.1 The dissipation due to normal motion of the grain boundary. This dissipation is due to attachment-detachment costs, with the mobility appropriate to the coupling factor $\beta_{i}$ (which $i$ to use will be decided as part of the minimization process). There is another issue here, because $v_{A}$ and $v_{B}$ are different. Since $A$ and $B$ are grains of the same material, we use the same mobility for crystals $A$ and $B$. For consistency with the non-rotating case and because we are counting the cost twice, we also introduce a factor of $\frac{1}{2}$. The dissipation function in the linear case becomes

$$
\int_{C_{o}} \frac{1}{2 M_{i}} \frac{v_{A}^{2}+v_{B}^{2}}{2} \mathrm{~d} s=\int_{C_{o}} \frac{1}{4 M_{i}}\left(\left(v_{\mathbf{n}}+\frac{V_{\perp}}{2}\right)^{2}+\left(v_{\mathbf{n}}-\frac{V_{\perp}}{2}\right)^{2}\right) \mathrm{d} s=\int_{C_{o}} \frac{1}{2 M_{i}}\left(v_{\mathbf{n}}^{2}+\frac{V_{\perp}^{2}}{4}\right) \mathrm{d} s .
$$

With non-linearity, the appropriate dissipation function is

$$
\int_{C_{o}} \frac{1}{2}\left(\int_{0}^{v_{\mathbf{n}}+V_{\perp} / 2} \mathcal{M}_{i}^{-1}(u) \mathrm{d} u+\int_{0}^{v_{\mathbf{n}}-V_{\perp} / 2} \mathcal{M}_{i}^{-1}(u) d u\right) \mathrm{d} s .
$$

3.3.2 The dissipation due to grain boundary sliding. Assuming $S$ is non-zero and obeys linear kinetics, the sliding velocity is $V_{\|}-\beta v_{\mathbf{n}}$ and the dissipation function for the sliding is

$$
\int_{C_{o}} \frac{1}{2 S}\left(V_{\|}-\beta_{i} v_{\mathbf{n}}\right)^{2} \mathrm{~d} s
$$

With non-linear sliding, the dissipation function is

$$
\int_{C_{o}} \int_{0}^{V_{\|}-\beta_{i} v_{\mathbf{n}}} \mathcal{S}^{-1}(u) \mathrm{d} u \mathrm{~d} s .
$$

In both cases, the choice of $i$ is part of the variational formulation.

If $S=0$ (or, in the non-linear case, $\mathcal{S}(u)=0$ for every $u$ ), then we require $V_{\|}-\beta_{i} v_{\mathbf{n}}=0$ at every point and do not have any sliding dissipation. 
3.3.3 The dissipation due to diffusion along the grain boundary. In the linear case, the dissipation function for diffusion, using equation (5), is

$$
\int_{C_{o}} \frac{1}{2 D} J^{2} \mathrm{~d} s=\int_{C_{o}} \frac{\dot{\theta}^{2}}{8 D}\left(R^{4}-\left(\overline{R^{2}}\right)^{2}\right) \mathrm{d} s,
$$

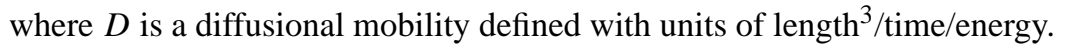

In the non-linear case, the dissipation function for diffusion is

$$
\int_{C_{o}} \int_{0}^{J} \mathcal{D}^{-1}(u) \mathrm{d} u \mathrm{~d} s
$$

\section{$3.4 \quad$ Putting them all together}

The case where $S=0$ (in the non-linear case, $\mathcal{S}=0$ ) must always be examined separately. We do not need to examine either $M=0$ or $D=0$ since either condition would prohibit all motion for a non-round initial grain boundary.

For linear kinetics with $S$ non-zero, by minimizing

$$
\int_{C_{o}}\left[\frac{\partial \gamma(\mathbf{n}, \theta)}{\partial \theta} \dot{\theta}-\kappa_{\gamma} v_{\mathbf{n}}+\frac{1}{2 M_{i}}\left(v_{\mathbf{n}}^{2}+\frac{v_{\perp}^{2} \dot{\theta}^{2}}{4}\right)+\frac{1}{2 S}\left(v_{\|} \dot{\theta}-\beta_{i} v_{\mathbf{n}}\right)^{2}+\frac{1}{2 D} j^{2} \dot{\theta}^{2}\right] \mathrm{d} s
$$

over all rotational rates $\dot{\theta}$ and all mean normal velocities $v_{\mathbf{n}}$ and possible coupling factors $\beta_{i}$ with associated mobilities $M_{i}$, we will determine all velocities, including $\dot{\theta}, v_{\mathbf{n}}, V_{\perp}$ and $V_{\|}$. Recall that all coefficients are allowed to depend on the normal direction of the interface at each point, via the inclination $\alpha$, as well as $\theta$.

Using non-linear kinetics with $\mathcal{S}$ non-zero, the expression to minimize is

$$
\begin{aligned}
\int_{C_{o}}\left\{\frac{1}{2} \int_{0}^{v_{A}} \mathcal{M}_{i}^{-1}(u) \mathrm{d} u\right. & \left.+\frac{1}{2} \int_{0}^{v_{B}} \mathcal{M}_{i}^{-1}(u) \mathrm{d} u+\int_{0}^{V_{\|}-\beta_{i} v_{\mathbf{n}}} \mathcal{S}^{-1}(u) \mathrm{d} u+\int_{0}^{J} \mathcal{D}^{-1}(u) \mathrm{d} u\right\} \mathrm{d} s \\
& +\int_{C_{o}}\left\{\frac{\partial \gamma}{\partial \theta} \dot{\theta}-\kappa_{\gamma} v_{\mathbf{n}}\right\} \mathrm{d} s .
\end{aligned}
$$

If $S=0$ (or, in the non-linear case, $\mathcal{S}=0$ ), we require

$$
v_{\|} \dot{\theta}-\beta_{i} v_{\mathbf{n}}=0
$$

and set $z_{i}=v_{\mathbf{n}} / \dot{\theta}=R^{2} /\left(\beta_{i} \sqrt{R^{2}+R^{\prime 2}}\right)$. In the linear case, we minimize

$$
\int\left(\frac{\mathrm{d} \gamma}{\mathrm{d} \theta} \dot{\theta}-\kappa_{\gamma} z_{i} \dot{\theta}+\frac{1}{2 M_{i}}\left(z_{i}^{2} \dot{\theta}^{2}+\frac{1}{4} v_{\perp}^{2} \dot{\theta}^{2}\right)+\frac{1}{2 D}\left(j^{2} \dot{\theta}^{2}\right)\right) \mathrm{d} s
$$

over all $\dot{\theta}$ and possible $i$. In the non-linear case, we minimize

$$
\begin{aligned}
\int_{C_{o}}\left\{\frac{1}{2} \int_{0}^{\left(z_{i}+\frac{1}{2} v_{\|}\right) \dot{\theta}} \mathcal{M}_{i}^{-1}(u) \mathrm{d} u+\frac{1}{2} \int_{0}^{\left(z_{i}-\frac{1}{2} v_{\|}\right) \dot{\theta}} \mathcal{M}_{i}^{-1}(u) \mathrm{d} u\right\} \mathrm{d} s \\
+\int_{0}^{j \dot{\theta}} \mathcal{D}^{-1}(u) \mathrm{d} u+\int_{C_{o}}\left\{\frac{\partial \gamma}{\partial \theta} \dot{\theta}-\kappa_{\gamma} v_{\mathbf{n}}\right\} \mathrm{d} s .
\end{aligned}
$$

over all $\dot{\theta}$ and possible $i$. 
It is possible to allow the choice of $\beta_{i}$ to vary from point to point, rather than using one choice for the whole curve. This issue is addressed in Sections 5.1 and 5.5.

\section{The general evolution laws}

\subsection{The case of no sliding}

When $S=0$, we can rewrite equation (10) as

$$
\beta_{i} \frac{\partial R}{\partial t}=-\dot{\theta} R
$$

by using the expressions for $v_{\mathbf{n}}$ and $V_{\|}$from (4). The minimum of expression (11) occurs at

$$
\dot{\theta}=\frac{\int\left(z_{i} \kappa_{\gamma}-\gamma^{\prime}\right) \mathrm{d} s}{\int\left(\left(z_{i}^{2}+v_{\perp}^{2} / 4\right) / M_{i}+j^{2} / D\right) \mathrm{d} s},
$$

for one or more values of $i$, where $z_{i}=R^{2} /\left(\beta_{i} \sqrt{R^{2}+R^{\prime 2}}\right)$ and $v_{\perp}$ and $j$ are as defined in (4) and 6). From this expression for $\dot{\theta}$ we compute $v_{\mathbf{n}}$ using (13). Finally, we need find the value(s) of $i$ which correspond to the smallest value of this minimum. Thus equations (13) and (14) are the rate laws for this case.

Quantity $[12]$ is minimized when

$$
\begin{aligned}
\frac{1}{2}\left(z_{i}-\frac{1}{2} v_{\perp}\right) \int_{C}\left(\mathcal{M}_{i}^{-1}\left(\left(z_{i}-\frac{1}{2} v_{\perp}\right) \dot{\theta}\right)+\right. & \frac{1}{2}\left(z_{i}+\frac{1}{2} v_{\perp}\right) \mathcal{M}_{i}^{-1}\left(\left(z_{i}+\frac{1}{2} v_{\perp}\right) \dot{\theta}\right) \mathrm{d} s \\
& +\int_{C} j \mathcal{D}^{-1}(j \dot{\theta}) \mathrm{d} s=\int_{C}\left(z_{i} \kappa_{\gamma}-\gamma^{\prime}\right) \mathrm{d} s .
\end{aligned}
$$

It is not obvious how to solve this for $\dot{\theta}$, nor that it determines a unique $\dot{\theta}$. Any $\dot{\theta}$ satisfying this equation has a corresponding $\dot{R}$ by $[13$.

\subsection{The case $S \neq 0$}

In the linear case, we minimize (8) to get the following coupled ODE and PDE:

$$
\begin{aligned}
& 0=\int_{C}\left(\frac{d \gamma}{d \theta}-\frac{\beta_{i}}{S} v_{\|} v_{\mathbf{n}}\right) \mathrm{d} s+\dot{\theta} \int_{C}\left(\frac{1}{4 M_{i}} v_{\perp}^{2}+\frac{1}{S} v_{\|}^{2}+\frac{1}{D} j^{2}\right) \mathrm{d} s, \\
& 0=-\kappa_{\gamma}-\frac{\beta_{i}}{S} v_{\|} \dot{\theta}+v_{\mathbf{n}}\left(\frac{1}{M_{i}}+\frac{\beta_{i}^{2}}{S}\right) .
\end{aligned}
$$

From (17p we get

$$
v_{\mathbf{n}}=\frac{M_{i} S}{S+\beta_{i}^{2} M_{i}}\left(\kappa_{\gamma}+\frac{\beta_{i}}{S} \frac{R^{2}}{\sqrt{R^{2}+R^{\prime 2}}} \dot{\theta}\right)
$$

Multiply 177 by $\frac{M_{i} \beta_{i}}{\beta_{1}^{2} M+S} v_{\|}$, integrate, solve for $\int_{C} \frac{\beta_{i}}{S} v_{\|} v_{\mathbf{n}} \mathrm{d} s$, and plug it into 16 to get

$$
\dot{\theta}=\frac{\int \frac{M_{i} \beta}{S+M_{i} \beta_{i}^{2}} \kappa_{\gamma} v_{\|} \mathrm{d} s-\int \gamma^{\prime} \mathrm{d} s}{\int \frac{1}{S+M_{i} \beta_{i}^{2}} v_{\|}^{2} \mathrm{~d} s+\int \frac{1}{4 M_{i}} v_{\perp}^{2}+\int \frac{1}{D} j^{2} \mathrm{~d} s} .
$$


In terms of $R$ and $R^{\prime}$, this is

$$
\dot{\theta}=\frac{\int \frac{M_{i} \beta}{S+M_{i} \beta_{i}^{2}} \kappa_{\gamma} R^{2} \mathrm{~d} \phi-\int \gamma^{\prime} \sqrt{R^{2}+R^{\prime 2}} \mathrm{~d} \phi}{\int \frac{1}{S+M_{i} \beta_{i}^{2}} \frac{R^{4}}{\sqrt{R^{2}+R^{\prime 2}}} \mathrm{~d} \phi+\int \frac{1}{4 M_{i}} \frac{R^{2} R^{\prime 2}}{\sqrt{R^{2}+R^{\prime 2}}} \mathrm{~d} \phi+\int \frac{1}{4 D}\left(R^{2}-\overline{R^{2}}\right)^{2} \sqrt{R^{2}+R^{\prime 2}} \mathrm{~d} \phi} .
$$

Therefore in the linear case, the rate laws are equations (18) and (19).

In the non-linear case, the scalar $\dot{\theta}$ and the function $v_{\mathbf{n}}$ must satisfy the following coupled equations:

$$
\begin{aligned}
0= & \int_{C}\left(\frac{d \gamma}{d \theta}+v_{\|} \mathcal{S}^{-1}\left(v_{\|} \dot{\theta}-\beta_{i} v_{\mathbf{n}}\right)+\frac{1}{2} v_{\perp} \mathcal{M}_{i}^{-1}\left(v_{\mathbf{n}}+\frac{1}{2} v_{\perp} \dot{\theta}\right)\right. \\
& \left.-\frac{1}{2} v_{\perp} \mathcal{M}_{i}^{-1}\left(v_{\mathbf{n}}-\frac{1}{2} v_{\perp} \dot{\theta}\right)+j \mathcal{D}^{-1}(j \dot{\theta})\right) \mathrm{d} s \\
0= & -\kappa_{\gamma}-\beta_{i} \mathcal{S}^{-1}\left(v_{\|} \dot{\theta}-\beta_{i} v_{\mathbf{n}}\right)+\frac{1}{2} \mathcal{M}_{i}^{-1}\left(v_{\mathbf{n}}+\frac{1}{2} v_{\perp} \dot{\theta}\right)+\frac{1}{2} \mathcal{M}_{i}^{-1}\left(v_{\mathbf{n}}-\frac{1}{2} v_{\perp} \dot{\theta}\right) .
\end{aligned}
$$

These follow directly from varying $\dot{\theta}$ and $v_{\mathbf{n}}$ in expression (8), using the fact that $\frac{\partial}{\partial v} \int_{0}^{g}(v) \mathcal{G}(u) \mathrm{d} u=\mathcal{G}(g(v)) \frac{\partial g}{\partial v}$. It is not easy to solve them to exhibit explicitly $\dot{\theta}$ and $v_{\mathbf{n}}$.

\section{Theorems}

\subsection{General results}

\subsubsection{Choice of $\beta_{i}$}

THEOREM 1 For a flat interface between grains with misorientation $\theta$ and inclination $\alpha$, when there is coupling and an imposed $V_{\|}$, then if there is an $i_{0}$ satisfying $M_{i_{0}}(\theta, \alpha) \beta_{i_{0}}^{2}(\theta)>$ $M_{i}(\theta, \alpha) \beta_{i}^{2}(\theta)$ for all $i \neq i_{0}$, then $v_{n}=V_{\|} / \beta_{i_{0}}$. If the maximum value of $M_{i} \beta_{i}^{2}$ is attained through the use of more than one $i$, then $v_{\mathbf{n}}$ is not uniquely determined, and any velocity in the interval between the corresponding $V_{\|} / \beta_{i}$ is possible.

Proof. The dissipation function per unit area for moving a flat interface by an imposed $v_{\|}$with coupling $\beta_{i}$ is $v_{\mathbf{n}}^{2} / M_{i}$, and $\beta_{i} v_{\mathbf{n}}=v_{\|}$. The energy change is zero; therefore the minimum occurs for all $i$ that produce the smallest value of $1 /\left(M_{i} \beta_{i}^{2}\right)$.

Comment 1 The value of $\theta$ where $M_{1} \beta_{1}^{2}=M_{2} \beta_{2}^{2}$ can depend on $\alpha$. Indeed, as discussed in the appendix, by symmetry it is known that for some $\theta$ for some crystals, $\beta_{2}$ is the correct choice at $\alpha=0$ and $\beta_{1}$ the correct choice at $\alpha=\pi / 4$.

Comment 2 Computations for circles with particular assumptions on the $\alpha$ dependence of $\left\{M_{i}\right\}$ indicate that the value of $\theta$ at which one $i$ ceases to be the uniquely best choice depends on the radius of the circle.

5.1.2 No sliding. Throughout this subsection, assume $\mathcal{S}(u)=0$ for every $u, \theta$ and $\alpha$; in the linear case, this means assume $S=0$.

THEOREM 2 The same $i$ (i.e. choice of coupling factor $\beta_{i}$ ) must be used at every point on $C$ for minimizing expression $[11]$. 
Proof. Because $v_{\mathbf{n}}$ is the normal velocity of the curve, it cannot be discontinuous. Because $V_{\|}-$ $\beta_{i} v_{\mathbf{n}}=0$ and $V_{\|}$is continuous (by (4p), the choice of $\beta_{i}$ must give a continuous function, and therefore the same $i$ must be chosen everywhere on the curve.

Theorem 3 Assume each $\beta_{i}$ depends only on $\theta$, not $\alpha$. Assume expression 111 is uniquely minimized using $i=i_{0}$ at $t=0$. Let

$$
f(\theta)=\exp \left(-\int_{\theta(0)}^{\theta} \frac{1}{\beta_{i_{0}}(\psi)} \mathrm{d} \psi\right) .
$$

Then $R(\phi, t)=f(\theta) R(\phi, 0)$ for all $t$ until such time that another value of $i$ also yields a minimum for expression (11). Therefore

(1) A non-circular initial grain boundary $C_{o}$ does not get rounder, and a circular $C_{o}$ stays circular.

(2) Relative to the crystal axes of $A$, the grain boundary shape rotates at the rate $\frac{1}{2} \dot{\theta}$.

Proof. We have assumed that for each $i, \beta_{i}$ is independent of $\alpha$, and from the previous theorem, we know that at each time, only one $i$ can be used for the entire curve. Thus we can rewrite equation (13) as

$$
\frac{\partial \ln R}{\partial t}=-\frac{\dot{\theta}}{\beta_{i}}
$$

and integrate to get

$$
R(\phi, t)=R(\phi, 0) f\left(\theta_{0}, \theta\right) .
$$

Given our choice of $\dot{\theta}_{A}=-\dot{\theta} / 2$, if we were to rotate the whole system at the rate $\dot{\theta} / 2$, then the lattice of $A$ would be fixed, the crystal $B$ would be rotating at the rate $\dot{\theta}$, and the shape would be rotating at the rate $\dot{\theta} / 2$. So we see that the shape rotates at half the rate of the lattice of $B$ relative to that of $A$, which is result (2).

Comment 3 The proof of (1) and (2) uses equation (13) alone, not (14).

Comment 4 For small $|\theta|, i=1, \beta_{1}$ is approximately $\theta$, and so $f(\theta) \approx \theta(0) / \theta(t)$.

Comment 5 We shall show, with more difficulty, that result (2) continues to hold when sliding is allowed, if there is a convenient shape attribute.

THEOREM 4 A family of curves $C(t)$ exists satisfying equations (13) and (14) and is uniquely determined up until at least the time when the expression (11) has the same minimum value for two choices of $i$. If equations 20 and 21 with $i=i_{0}$ have a unique solution for $\dot{\theta}$, then the same results hold for those equations.

Proof. When $R(\phi, t)=f(\theta) R(\phi, 0)$ is plugged into 19 , one obtains an equation for $\dot{\theta}$ depending only on $\theta$. This is an autonomous first order differential equation for the scalar $\theta$ and has a unique solution. In the non-linear case, plugging $R(\phi, t)=f(\theta) R(\phi, 0)$ into (15) with $i=i_{0}$ gives an implicit equation for $\dot{\theta}$. If it is solved for $\dot{\theta}$, then the resulting equation can be integrated to get $\theta(t)$.

Comment 6 The behavior when two different $i$ 's give the same minimum has not yet been investigated. See Section 5.5 concerning the consequences of there being more than one $\beta_{i}$. 
5.1.3 With sliding. Existence and uniqueness for the linear and non-linear non-zero- $S$ equations have not yet been investigated. The conclusions of the following theorem follow directly from examining the rate laws (18) and (19):

THEOREM 5 (1) The rate laws for $S=0$ are the limits of the rate laws as $S$ approaches zero.

(2) Suppose $C_{o}$ has a plane of mirror symmetry; without loss of generality, we may assume it is $\phi=0$, so that $R$ is an even function of $\phi$. Assume $\gamma, M, S$, and $\beta$ are even functions of $\alpha$, where $\alpha$ is measured from the ray $\phi=0$ (in our doubly rotating setup). Then $v_{\mathbf{n}}$ is also an even function of $\phi$, by $(18)$, and therefore at later times $\phi=0$ is again a mirror. Since $\dot{\theta}_{A}=-\dot{\theta}_{B}$, the rate of rotation of the mirror relative to the axes of crystal $A$ is half that of the rotation of crystal $B$ relative to crystal $A$.

Comment 7 If $R^{\prime}$ is zero for all $\phi$ and if $\gamma, M_{i}$, and $S$, as well as $\beta_{i}$, are independent of $\alpha$, the rate laws are the equations (1) for circles studied in [5].

\subsection{Slightly perturbed circles, linear kinetics}

There are several insights easily obtained from the nearly circular case $R(0)=r(1+\epsilon \cos (m \phi))$. (The restriction that $m \geqslant 2$ occurs because $m=0$ gives a circle and $m=1$ gives an off-center circle. A more general perturbed shape would have sine and cosine terms; with cosine terms only the shape is mirror symmetric about $\phi=0$.) Assume all coefficients are independent of $\alpha$. Changing $\epsilon$ to $-\epsilon$ simply rotates the initial grain shape. Since independence of $\alpha$ is assumed, this should not change the kinetics. The rates of shrinking and rotation should be even functions of $\epsilon$, since changing the sign of $\epsilon$ just rotates the original shape. The rate of approach to a circular shape $-\dot{\epsilon}(t)$ should be an odd function of $\epsilon$, for the same reason. Thus to first order in $\epsilon$ there is no reduction in either the rate of shrinking or the rate of rotation.

We therefore expect that such small perturbations of a circle should have little effect. We explore what those effects are.

5.2.1 Case $S=0$. Because the shape remains the same when $S=0$, we only have to investigate the effect of the perturbations on $\dot{\theta}$ and $\dot{r}$; $\epsilon$ will remain constant and no new harmonics will appear. Also, $\dot{r}=-\dot{\theta} r / \beta$, so $\dot{\theta}$ determines $\dot{r}$.

Using equation (14) and keeping terms up to order $\epsilon^{2}$, we compute

$$
\dot{\theta}=\frac{\beta_{i} M_{i h}\left(\gamma+\frac{\partial^{2}}{\partial \alpha^{2}} \gamma-\beta_{i} \gamma^{\prime}\right)}{r^{2}} \frac{1+\epsilon^{2} \frac{m^{2}}{4}}{1+\epsilon^{2}\left(\frac{m^{2} \beta_{i}^{2}-2 m^{2}+12}{8}+\frac{M_{i h} \beta_{i}^{2} r^{2}}{2 D_{h}}\right)}
$$

where $M_{i h}$ and $D_{h}$ are the harmonic averages defined by $2 \pi / M_{i h}=\int_{0}^{2 \pi}\left(1 / M_{i}\right) \mathrm{d} \alpha$ and $2 \pi / D_{h}=$ $\int(1 / D) \mathrm{d} \alpha$.

Computational Result 6 For $R(0)=r(1+\epsilon \cos (m \phi))$ and small enough $\epsilon$, the rotation rate at $t=0$ is indeed within order $\epsilon^{2}$ of that for a circle, provided $M_{i} \beta_{i}^{2} r^{2} / D_{h}$ is of order 1 for any minimizing $i$. If it is much less than 1 , surface diffusion has negligible effect; if it is of order 1 , then both $M_{i h}$ and $D_{h}$ play a role. If, however, $M_{i h} \beta_{i}^{2} r^{2} / D_{h} \gg \epsilon^{-2} \gg 1$, then we obtain

$$
\dot{\theta}=\frac{2 D_{h}}{\beta_{i} r^{4} \epsilon^{2}}\left(\gamma+\frac{\partial^{2}}{\partial \alpha^{2}} \gamma-\beta \gamma^{\prime}\right)
$$


and surface diffusion entirely dominates the rotation rate, which need not be at all close to that for circles.

5.2.2 Case $S \neq 0$. With the results for $S=0$ in mind, we assume that $\epsilon^{2}$ is small enough compared to appropriate ratios of kinetic coefficients that all terms involving $\epsilon$ and $\epsilon^{2}$ are in fact much less than 1, and in particular that $\epsilon^{2} \ll D /\left(r^{2} \beta_{i}^{2} M_{i}\right)$ at all $\alpha$. Since the situation is already complicated, we assume that none of $\gamma, M_{i}, S$, and $D$ depend on $\alpha$.

By equation [19],

$$
\dot{\theta}=\frac{\beta M\left(\gamma-\left(\beta+\frac{S}{M \beta}\right) \gamma^{\prime}\right)}{r^{2}}\left(1+\epsilon^{2}\left(\frac{m^{2} \beta^{2}-2 m^{2}+12}{8}+\frac{M \beta^{2} r^{2}}{2 D}+\frac{S m^{2}}{8 M}+\frac{S r^{2}}{2 D}\right)\right) .
$$

If we write $R(t)=\left(r+r_{1}(t)\right)\left(1+\left(\epsilon+\epsilon_{1}(t)\right) \cos (m \phi)+h_{1}(t) \cos (2 m \phi)\right)+E(t)$, where $E$ is all the other terms and $r_{1}(0)=\epsilon_{1}(0)=h_{1}(0)=E(0)=0$, then

$$
\dot{R}(0)=\dot{r}_{1}(1+\epsilon \cos (m \phi))+r \cos (m \phi) \dot{\epsilon}_{1}+r \cos (2 m \phi) \dot{h}_{1}+\dot{E} .
$$

We can write $\dot{R}$ (from equation (18) in a way so as to identify $\dot{r}_{1}, \dot{\epsilon}_{1}, \dot{h}_{1}$, and $\dot{E}$ :

$$
\begin{aligned}
\dot{R}= & -\left(M \frac{\gamma-\beta \gamma^{\prime}}{r}+\frac{Z}{8 D r\left(\beta^{2} M+S\right)} \epsilon^{2}\right)(1+\epsilon \cos (m \phi)) \\
& +r \cos (m \phi)\left(-\epsilon \frac{M S \gamma\left(m^{2}-2\right)}{r^{2}\left(\beta^{2} M+S\right)}\right) \\
& +r \cos (2 m \phi)\left(\epsilon^{2} \frac{\left(3 m^{2}-1\right) \gamma D M S}{2 D r^{2}\left(\beta^{2} M+S\right)}\right)+O\left(\epsilon^{3}\right)
\end{aligned}
$$

where

$$
\begin{aligned}
Z= & \beta^{4} M^{2}\left(m^{2} D+4 M r^{2}\right)\left(-\gamma+\beta \gamma^{\prime}\right)-\left(4 m^{2}-4\right) \gamma D M S \\
& +\left(\left(4 m^{2}-12\right) D M-m^{2} D S-4 M S r^{2}\right)\left(\beta^{2} \gamma M-\beta \gamma^{\prime} S\right) \\
& +2 \beta^{3} \gamma^{\prime} M\left(m^{2} D S+4 M r^{2} S-\left(2 m^{2}-6\right) D M\right) .
\end{aligned}
$$

Computational Result 7 To first order, the rates of change of $r+r_{1}(t)$ and $\theta(t)$, namely $\dot{r}_{1}$ and $\dot{\theta}$, are the same as those for circles as given in equation 11 . The second order terms for $\dot{r}_{1}$ and $\dot{\theta}$ can be positive, negative, or zero depending on the given $S, D, M, \gamma$ and $\beta$ (all non-zero) as well as the geometric variables $m$ and $r$. To second order in $\epsilon$, only the $\cos (2 m \phi)$ harmonic appears, and its coefficient is positive, as it must be. The rate of change of the perturbed shape toward circular, given by $\dot{\epsilon}$, is negative, as expected, and does not depend on $D$ to third order in $\epsilon$. The implication is that differences in the rate of lateral displacement from coupled motion driven by gradients in curvature are accommodated primarily by sliding and not by diffusion; this agrees with the $S=0$ result of no shape change. As would be expected, the rate of shrinking increases with decreasing distance between features: it depends directly on $m^{2}-2$ and inversely on $r^{2}$. When $\beta^{2} M / S \gg 1$, the rate ceases to depend on $M$ and becomes linear in $S$; conversely, when $\beta^{2} M / S \ll 1$, the rate is controlled by $M$, and ceases to depend on either $S$ or $\beta$. 


\subsection{Grains with high aspect ratios, linear kinetics}

In this section, we assume that none of the parameters depend on $\alpha$ (i.e., everything is independent of the normal to the grain boundary). Therefore these results would need to be re-examined when $\theta$ is close to the interval where the choice of $\beta_{i}$ varies with $\alpha$ for flat interfaces, in the case where more than one $\beta_{i}$ is geometrically possible.

Suppose the grain boundary consists of two line segments parallel to the $x$ axis, one a distance $\epsilon$ below it and one a distance $\epsilon$ above it and each extending from $x=-a$ to $x=a$, together with semicircular caps at each end. To compare with the case of an approximation to a circle of radius $r$ we should assume $4 \epsilon a=\pi r^{2}$ as well as $\epsilon \ll a$.

The mean square radius is essentially $\int_{-a}^{a} x^{2} d x / 2 a=a^{2} / 3$. The denominator of $\dot{\theta}$ in equation (19) is, to order $\epsilon$,

$$
\epsilon a^{2} \pi / 4+\frac{S+\beta^{2} M}{4 M}\left(\frac{4}{3} a^{3}+\pi a^{2} \epsilon\right)+\frac{S+\beta^{2} M}{4 D}\left(\frac{16}{45} a^{5}+\frac{16}{9} \pi \epsilon a^{4}\right) .
$$

The numerator is $4 a\left(M \beta \gamma-M \beta^{2} \gamma-S \gamma^{\prime}\right)$, leading to

$$
\dot{\theta}=\frac{M \beta \gamma-\beta^{2} M \gamma^{\prime}-S \gamma^{\prime}}{a^{2}\left(S+\beta^{2} M\right)\left(\frac{1}{12 M}+\frac{a^{2}}{45 D}\right)}
$$

to zeroth order in $\epsilon$. Similar expressions are expected for other high-aspect ratio grains.

Computational Result 8 If $D$ is much less than $\frac{12}{45} a^{2} M$, then

$$
\dot{\theta} \approx \frac{45 D}{a^{4}}\left(\frac{M \beta}{S+\beta^{2} M}-\gamma^{\prime}\right) .
$$

Thus in this case the rotation rate is controlled by diffusion, and if $D a^{-4}$ is very small, that rotation rate is very small. In particular, it is slower than that of the circle with area $\pi r^{2}$ equal to $4 a \epsilon$ if $D<\left(S+M \beta^{2}\right) r^{6} / 45$.

It is therefore reasonable to assume as an approximation to this case that $\dot{\theta}=0$. When sliding is easy ( $S$ is large compared to $\beta^{2} M$ ), we have motion by weighted curvature with mobility,

$$
v_{\mathbf{n}} \approx M \kappa_{\gamma} .
$$

On the other hand, when $S \beta^{-2}$ is intermediate between $M$ and $a^{2} D$, then

$$
v_{\mathbf{n}}=\frac{S}{\beta^{2}} \kappa_{\gamma} .
$$

This is also a version of motion by weighted curvature, but the "mobility" is not determined by attachment-detachment kinetics but rather by sliding and coupling.

COMMENT 8 In [8], a different motion was considered, where $\gamma$ itself, rather than $\int_{C} \gamma \mathrm{d} s$, was required to decrease and there was no coupling, and it considered polycrystals. That paper also found that far-from-round crystals shrank at a rate inversely proportional to $a^{4}$.

\subsection{Qualitative picture}

We now have a qualitative picture of what happens, for an initial shape that is relatively large and not very round when $S$ may be very small but is not zero and the initial misorientation is not large: the motion is by weighted curvature, with the rate depending on the relationship between $M \beta^{2}$ and $S$, 
and with the misorientation not changing. In either case, crystal $B$ shrinks and becomes rounder. Then the diffusion becomes less of a hindrance, and the crystal begins to rotate at an appreciable rate, and all kinetic factors are important.

Conjecture: If all parameters are independent of $\alpha$, then we expect a grain of arbitrary initial shape to become approximately round as it shrinks to a point.

\section{Some open problems}

Various constraints that were made in this paper should be relaxed. In particular, the restriction that the center of rotation is fixed ought to be removed, and bulk driving forces as well as external stresses could be added, as in [5]. The requirement of smoothness for $\gamma$ might be lifted; it would be interesting to see the effects of shape accommodation at the opposite extreme where the Wulff shape is polyhedral.

More ambitiously, three-dimensional embedded crystals should be studied. Boundaries between two three-dimensional crystals are characterized by five angles, three to specify the relative misorientation, and two for the orientation of the plane; $\gamma$ is a scalar function of these angles, while other quantities, such as $\beta$, are tensors.

Also, an attempt might be made to use statistical mechanics to connect the molecular dynamics simulations to the continuum model proposed. Within the continuum model, it is possible that the whole variational problem could be set up in terms of finite time steps; this would be particularly useful when using $\gamma$ with fewer restrictions and in higher dimensions, where singularities can develop. See [1] for the possibilities; there one minimizes with $\Delta t>0$ (and no coupling or its associated shape accommodation) to obtain approximate flows and then finds a limit of the approximate flows.

Mathematical questions remaining in the problem studied in this paper range from proving existence and uniqueness of solutions in the fully non-linear case to determining whether embedded grains shrink to round points. One might also try to determine whether there are any features that continue to hold approximately for an evolving grain shape without a mirror symmetry, in analogy to the conclusion that when there is a mirror symmetry plane initially, a mirror symmetry plane continues to exist and rotates at half the rate of the change in misorientation.

Many issues will arise when attempting to include coupling and shape accommodation in polycrystals, with their triple and higher junctions between neighboring grains. As a specific question, consider a two-dimensional polycrystalline microstructure and focus on one shrinking "equi-axed" grain surrounded by, say, three other grains and thus having three triple points; what are the equations of motion, and do our results for slightly perturbed circles hold?

The major materials science question is: what is the effect of coupling on the many processes in which grain boundary motion is a component, such as grain growth, discontinuous precipitation, diffusion induced grain boundary motion, etc.? These are complex processes even without coupling. Grain boundary properties show enormous variations with the five angles which characterize them, and shape accommodation is a difficult problem. Triple junctions play major roles which have received little attention [8].

\section{Acknowledgments}

We are most grateful to S. G. Srinivasan and Yuri Mishin for permission to use results from their as yet unpublished simulations, and to J. A. Warren and G. McFadden for critical reviews. 


\section{Appendix: Further information about the coupling factor $\beta$ for square symmetry crystals, 3-d models, and simulations}

In this appendix, we consider planar grain boundaries. We assume the reference lattice for the two crystals in the bicrystal has square $(4 \mathrm{~mm})$ symmetry, with the sides of the square aligned with the coordinate axes. We orient the bicrystal in laboratory coordinates so that crystal $B$ is rotated by an angle between 0 and $\pi / 2$ and crystal $A$ by its negative; we call this angle $\theta / 2$, so that $\theta$ is a misorientation angle and the laboratory vector $(1,0)$ bisects it. The inclination $\alpha$ is chosen to be the angle of the grain boundary from $(1,0)$, i.e., the angle from $(0,1)$ to the oriented normal from crystal $A$ to crystal $B$, measured counterclockwise. Note that all physical properties of the boundary are periodic in $\theta$, of period $\pi$, and in $\alpha$, of period $\pi / 2$.

When $\theta=0$, the two crystals are in alignment. When $\theta=\pi / 2$, the two crystals (one rotated by $\pi / 4$ and the other by $-\pi / 4$ from their reference crystal) are again in alignment. When $\alpha=0$, these two types of alignment give rise to two different kinds of symmetric low-angle boundaries for misorientations near them. The disappearing low-angle boundary in the $\theta \rightarrow 0$ limit is aligned with the $(1,0)$ vector in the reference crystal, while in the $\theta \rightarrow \pi / 2$ limit it is aligned with the $(1,1)$ diagonal of the reference crystal. Low angle boundaries are composed of widely separated dislocation, and in these two limits the dislocations have different Burgers vectors and different energies and their motion differs in sign and in the deformation that results.

If $\theta<\pi / 2$, then the line at angle $\pi / 4$ is a bisector of the complement of $\theta$, the angle from the ray at $\theta / 2$ to the ray at $\pi / 2-\theta / 2$. If we rotate the entire bicrystal by $-\pi / 4$, then the angle $\alpha$ is taken to $\alpha-\pi / 4$, the orientation of crystal $A$ becomes $-(\theta / 2+\pi / 4)$, and the orientation of crystal $B$ becomes $\theta / 2-\pi / 4$, which is equivalent by the square symmetry to $\theta / 2+\pi / 4$. Thus, except for its position in laboratory coordinates, the bicrystal described by $(\theta, \alpha)$ is identical to the bicrystal described by $(\theta+\pi / 2, \alpha-\pi / 4)$. If there are no laboratory fields that interact differently with different orientations, then all physical properties should be the same for $(\theta, \alpha)$ and for $(\theta+$ $\pi / 2, \alpha-\pi / 4)$.

This same rotation by $-\pi / 4$ takes the ray at angle $\pi / 2-\theta / 2$ to $\pi / 4-\theta / 2$, and that angle $\theta / 2$ to $-(\pi / 4-\theta / 2)$. Thus, except for orientation in laboratory coordinates, the bicrystal described by $(\theta, \alpha)$ is identical to that described by $(\pi / 2-\theta, \alpha-\pi / 4)$. In the field-free case, whether $\theta$ or its complement is chosen as the misorientation does not matter as long as $\alpha$ is incremented by $\pi / 4$ for the latter compared to the former. For every given $\theta$, there are two kinds of symmetric boundaries, $\alpha=0$ and $\alpha=\pi / 4$ (and their equivalents under rotation by $\pi / 2$ ); the $(\theta, \pi / 4)$ symmetric bicrystal is equivalent to the $(\pi / 2-\theta, 0)$ symmetric bicrystal.

The same symmetries occur for a three-dimensional bicrystal restricted to have a common cube axis; the two angles have the same definitions and invariances.

In this field-free case, we may therefore restrict consideration to the case $0<\theta<\pi / 2$ and $0 \leqslant \alpha<\pi / 4$, as all other bicrystals are related to such a pair by the symmetries discussed.

By simple shears, two types of ideal motion are geometrically possible over the entire $\theta$ and $\alpha$ range:

$$
\beta_{1}(\theta, \alpha):=2 \tan (\theta / 2)>0, \quad \beta_{2}(\theta, \alpha):=2 \tan \left(\frac{1}{2}\left(\theta-\frac{\pi}{2}\right)\right)<0,
$$

with $\beta_{1}$ easier to accomplish near $\theta=0$ and (by symmetry) $\beta_{1}$ easier to accomplish near $\theta=\pi / 2$. (Two other crystallographically possible values of $\beta, \beta_{3}(\theta, \alpha):=2 \tan \left(\frac{1}{2}(\theta-\pi)\right.$ ) and $\beta_{4}(\theta, \alpha):=$ $2 \tan \left(\frac{1}{2}\left(\theta-\frac{3 \pi}{2}\right)\right)$, are so much more difficult to achieve that they can be ignored, as long as we use symmetry and take $\theta$ in the interval $(0, \pi / 2)$.) 


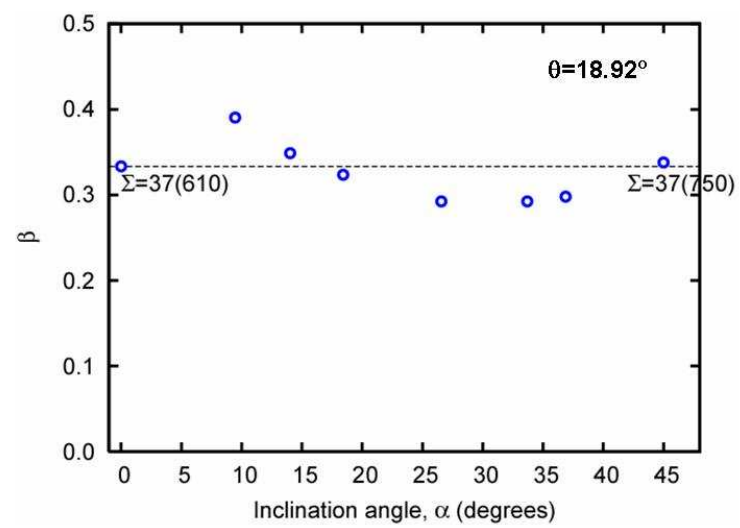

FIG. 4. Inclination (normal) dependence of $\beta$ (Yuri Mishin data). Unpublished data [9] from MD simulations indicating that for $\theta \approx 19^{\circ}, \beta_{1}$ is an approximately constant function of $\alpha$. There is no jump to $\beta_{2}$.

The simulations in 3D are consistent with these symmetries [3]. For $\alpha=0$ and not too high a temperature, they reveal accurate agreement with $\beta=\beta_{1}$ for $0<\theta \lesssim 0.2 \pi$ and $\beta=\beta_{2}$ for $0.2 \pi \lesssim \theta<\pi / 2$, with what seems to be a discontinuity at $\theta \approx 0.2 \pi$ (see Figure 2). Similarly, for $\theta$ near zero, the relative motion is approximately constant with $\beta=\beta_{1}$ for all $\alpha$ (see Figure 4); lack of exact agreement might be due to interactions between dislocations.

Symmetry does not dictate at which $\theta$ the observed $\beta$ should switch from $\beta_{1}$ to $\beta_{2}$. Symmetry does say that because it is observed to occur at $\approx 0.2 \pi$ when $\alpha=0$, it should occur at $\approx(0.5-0.2) \pi$ when $\alpha=\pi / 4$. For $\theta$ between approximately $0.2 \pi$ and $0.3 \pi$ a change of sign in the observed $\beta$ must occur between $\alpha=0$ and $\pi / 4$, because $\beta(\theta, 0)=\beta_{2}(\theta, 0)$ and $\beta(\theta, \pi / 4)=\beta_{1}(\theta, 0)$ for such $\theta$. This is observed, but in a rather unexpected fashion. Where a jump between $\beta=\beta_{2}(\theta, \alpha)$ and $\beta=\beta_{1}(\theta, \alpha)$ might be expected when $\theta$ is fixed at $2 \arctan (1 / 2) \approx 37^{\circ}$ (a special grain boundary, called $\Sigma 5$ in the materials science literature; see e.g. [3]) as $\alpha$ is varied, each of those being essentially independent of $\alpha$, instead the ratio $V_{\|} / v_{\mathbf{n}}$ appears to diverge; see Figure 5a. Plotting

(a)

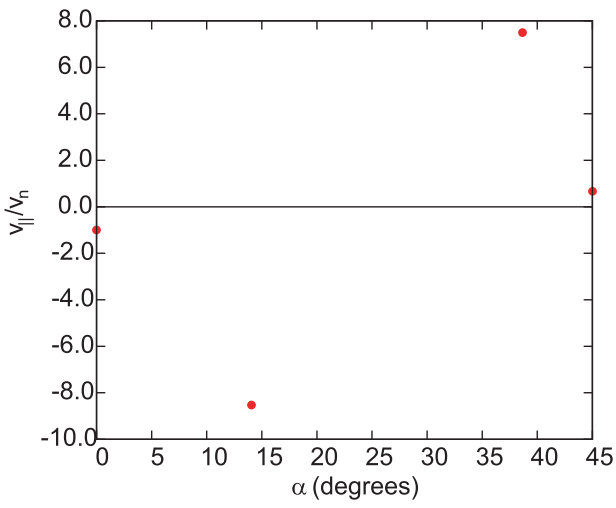

(b)

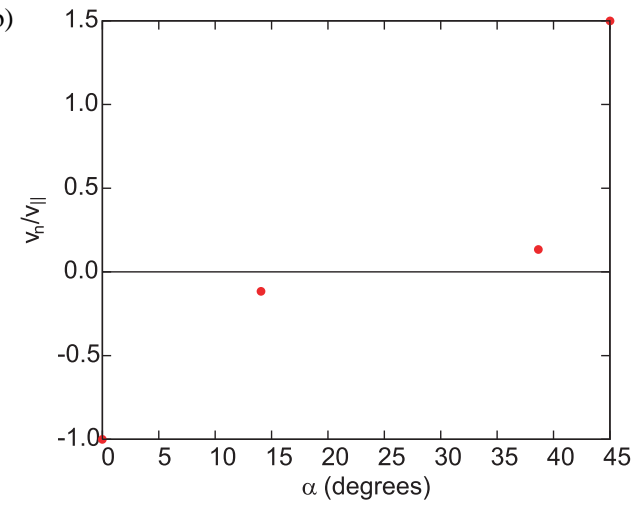

FIG. 5. Unpublished preliminary data [9] from MD simulations indicating that for $\theta \approx 37^{\circ}$, there is a change from positive to negative motions with increasing $\alpha$. There are only four points, the temperature is fairly high, and the error bars would be large. This change is discussed in this appendix and appears to involve a jump from $-\infty$ to $+\infty$, though it might be from a finite negative value to a finite positive value. In (b), $v_{\mathbf{n}} / V_{\|}$is instead plotted, useful for comparing with sliding behavior. 
$v_{\mathbf{n}} / V_{\|}$as in Figure $5 \mathrm{~b}$ shows that the normal velocity becomes almost uncoupled from the tangential. A possible explanation is sliding. Sliding increases $v_{\|}$without adding to $v_{\mathbf{n}}$. Were it not for its characteristic behavior in the simulations (as in Figure 3), its presence would seem like an increase in $|\beta|$, with pure sliding looking like $|\beta|=\infty$.

At very low temperatures, motion of perfect periodic symmetric flat grain boundaries is difficult to initiate, and at $\alpha=0$ initiating motion using the dislocations for $\beta_{1}$ is more difficult than using those for $\beta_{2}$. In the simulations stress built up until motion occurred, and the motion was by stickslip. The stress for $\beta_{1}$ was greater than for $\beta_{2}$ [3]; for small $\theta$, with the grains forced to translate, the motion often initiated with $\beta_{2}$. But once motion was initiated and the boundary became less regular, sometimes part of a boundary would suddenly begin to move with $\beta_{1}$ while the rest moved with $\beta_{2}$; eventually it would all move with $\beta_{1}$. The relevance of this for more arbitrary boundaries is unclear, and will be neglected in this paper: if $\beta_{1}$ turns out to be the variationally preferred coupling, then we will assume the system finds a way to accomplish it. Since in this paper we deal with curved boundaries, these issues should not be so important.

At intermediate temperatures, motion in the simulations changes from stick-slip to being more continuous. At higher temperatures (the temperature depending on $\theta$ ), sliding begins to occur [4]; see Figure 3.

\section{REFERENCES}

1. Almgren, F., Taylor, J. E., \& Wang, L. H. Curvature driven flows: A variational approach. SIAM J. Control Optim. 31 (1993), 387-438. Zbl 0783.35002 MR 1205983

2. Cahn J. W., \& TAYlor J. E. Surface motion by surface diffusion. Acta Metall. Mater. 42 (1994), 1045-1063.

3. Cahn, J. W., Mishin, Y., \& Suzuki, A. Duality of dislocation content of grain boundaries. Philos. Mag. 86 (2006), 3965-3980.

4. Cahn, J. W., Mishin, Y., \& Suzuki, A. Coupling grain boundary motion to shear deformation. Acta Mater. 54 (2006), 4953-4975.

5. CAHn, J. W., \& TAYLOR, J. E. A unified approach to motion of grain boundaries, relative tangential translation along grain boundaries, and grain rotation. Acta Mater. 52 (2004), 4887-4898.

6. CARTER, W. C., TAYlor, J. E., \& CAHN, J. W. Variational methods for microstructural evolution, JOM 49 (1997), no. 12, 30-36.

7. Goldstein, H. Classical Mechanics. Addison-Wesley, Reading, MA (1951). Zbl 0043.18001 MR 0043608

8. HARRIS, K. E., Singh, V. V., \& KING, A. H. Grain rotation in thin films of gold. Acta Mater. 46 (1998), 2623-2633.

9. Mishin, Y. Private communication (2006).

10. SRinivasan, S. G. Unpublished results.

11. SRinivasan, S. G., \& CAHn, J. W. Challenging some free-energy reduction criteria for grain growth. Science and Technology of Interfaces, S. Ankem et al. (eds.), TMS, Warrendale, PA (2002), 3-14.

12. TAYlor, J. E. Dissipation and the Allen-Cahn and Cahn-Hilliard equations. Preprint.

13. TAYlOR, J. E., \& CAHN, J. W. Linking anisotropic sharp and diffuse surface motion laws via gradient flows. J. Statist. Phys. 77 (1994), 183-197. Zbl 0844.35044 MR 1300532

14. TAYlor, J. E., Handwerker, C. A., \& CAHN, J. W. Geometric models of crystal growth. Acta Metal. Mater. 40 (1992), 1443-1474.

15. Warren, J. A., Kobayashi, R., Lobkovsky, A. E., \& Carter, W. C. Extending phase field models of solidification to polycrystalline materials. Acta Mater. 51 (2003), 6035-6058. 